\title{
INFRARED SPECTROSCOPY AND MAPPING OF IC443
}

\author{
Gillian S. Wright ${ }^{1,2}$, James R. Graham ${ }^{3}$, \& Andrew J. Longmore ${ }^{2}$ \\ ${ }^{1}$ United Kingdom Infrared Telescope, ${ }^{2}$ Royal Observatory, Edinburgh, \\ ${ }^{3}$ Lawrence Berkeley Laboratory, University of California
}

\begin{abstract}
We present infrared (IR) spectroscopy and imaging of the supernova remnant IC443. [FeII] $1.644 \mu \mathrm{m}$, Brackett $\gamma$ and $\mathrm{H}_{2} 1-0 \mathrm{~S}(1)$ were detected. The most striking feature of the spectra is the high [FeII] $1.644 \mu \mathrm{m} /$ Brackett $\gamma$ ratio, which is typically $\sim 30$. We argue that this ratio is due to shock excitation. Two 5 ' $\times 3$ ' maps of the remnant in the $1.644 \mu \mathrm{m}$ [FeII] line are used to study the line excitation. The IR [FeII] line is a sensitive probe for regions of shocked gas which is especially useful where the extinction is high, or the shocks are too slow to excite optical lines.
\end{abstract}

\section{INTRODUCTION}

Optical spectroscopy is a well established tool for the study of fast shocks $(\sim 100 \mathrm{~km} / \mathrm{s})$. However, a low excitation IR line which suffers less extinction than optical lines, and falls in one of the near $I R$ windows would be a valuable shock diagnostic. The forbidden IR lines arising from the low lying levels of $\mathrm{Fe} I I$ satisfy these critera.

The SNR IC443 is an excellent object for developing IR spectroscopy as a shock diagnostic since the blast wave is interacting with interstellar material of a variety of densities at different positions around the SNR shell. This paper summarises the first stage of our study of IC443 in which the $1.644 \mu \mathrm{m}$ [Fe II] line is reported in fast optically bright shocks (see also Graham, Wright \& Longmore 1987).

\section{OBSERVATIONS}

The data were obtained at UKIRT using the circular variable filter $(R \sim 120)$ in the common user IR photometer UKT9. [FeII] emission line maps of the NE rim and the E extremity of the SNR were made with the CVF centered on $1.644 \mathrm{~mm}$, using a $20^{\prime \prime}$ aperture sampled on a $15^{\prime \prime}$ grid. Since the remnant is large $\left(1^{\circ}\right)$ we chopped $3^{\prime}$ off the edge of the remnant but did not beamswitch. Figs. 1 \& 2 show the continuum subtracted maps.

\section{Figure 1 \\ An [FeI] $1.644 \mu \mathrm{m}$ image of the NE section of IC443. The contours start at 60 above the noise, and go up in units of $6 \sigma$ linearly. The map is 3'x5.5'. The sharp edge of the remnant can clearly be seen, and a number of bright knots are evident.}

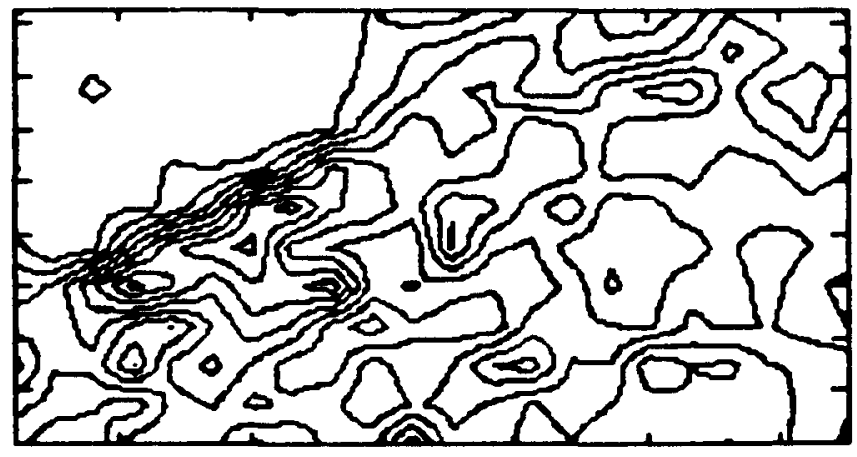

Spectra were measured on the optically bright NE rim of the remnant (posn. A and 1) and at the E edge (posn. 31). The spectra were obtained in a $20^{\prime \prime}$ aperture using a chop of 1.5-3'. Fig. 3 shows the spectrum at position 1. 


\section{LINE IDENTIFICATION}

At positions $A$ and 1 to the NE we detected lines at $1.644 \mu \mathrm{m}$ and $2.165 \mu \mathrm{m}$; identified as [FeII] $1.6440 \mu \mathrm{m}$ and $\mathrm{Br} \gamma(\lambda=2.1655 \mu \mathrm{m})$. At position 31 we detected the [FeII] line and a line at $2.121 \mu \mathrm{m}$ which is the 1-0 S(1) transition of molecular hydrogen.

Figure 2

An $[\mathrm{FeII}] 1.644 \mu \mathrm{m}$ image of the eastern edge of IC443. The edge of the remnant is clearly visible. The map dimensions are $2.75 ' x 5.25$ '. The contours are the same as in Fig. 1.

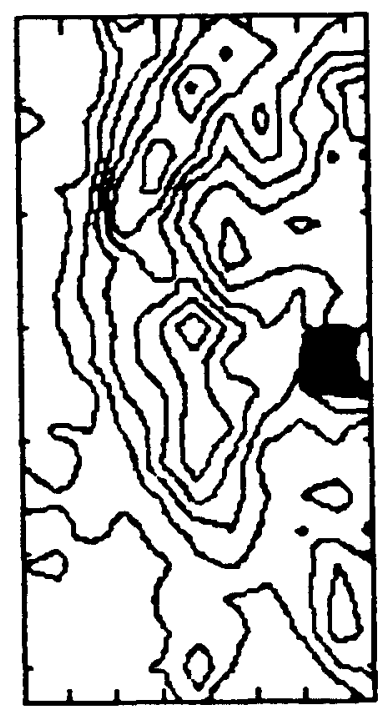

\section{IR IMAGES}

The [FeII] $1.644 \mu \mathrm{m}$ line maps of the optically emitting shocks in IC443 (Fig. 1), demonstrate the ubiquitous nature of this emission. There is a broad correlation between the [FeII] and $\mathrm{H} \alpha$ (Fesen \& Kirshner 1980) emission, although close inspection reveals several knots which are much more prominent in the $[\mathrm{FeI}]$ map, indicating large variation of the ratio [FeII]/H $\alpha$ on small spatial scales. Our map of the eastern section (15'SE of the northern map) shows a much higher mean $[\mathrm{FeI}] / \mathrm{H} \alpha$ ratio, indicating a significant large scale gradient across the remnant. Comparison of our maps with images in lines other than $\mathrm{H} \alpha$ (Hester, 1987 and private communication) indicates even greater line ratio variations. For example, [OIm] $5007 \AA$ and [FeI] are both bright in the region covered by Figure 1, but the [OIII] emission associated with Figure 2 is virtually undetectable even though [FeII] is still almost as bright as it is in Fig. 1a suggesting that [OII] $0.5007 \mu \mathrm{m} /[\mathrm{FeI}] 1.644 \mu \mathrm{m}$ may be a sensitive shock speedometer.

Figure 3

An IR spectrum of IC443 on the NE part of the SNR. The wavelengths of $[\mathrm{FeII}]$ $1.644 \mu \mathrm{m}$ and $\mathrm{Br} \gamma$ are indicated. The wavelength scale is not continuous.

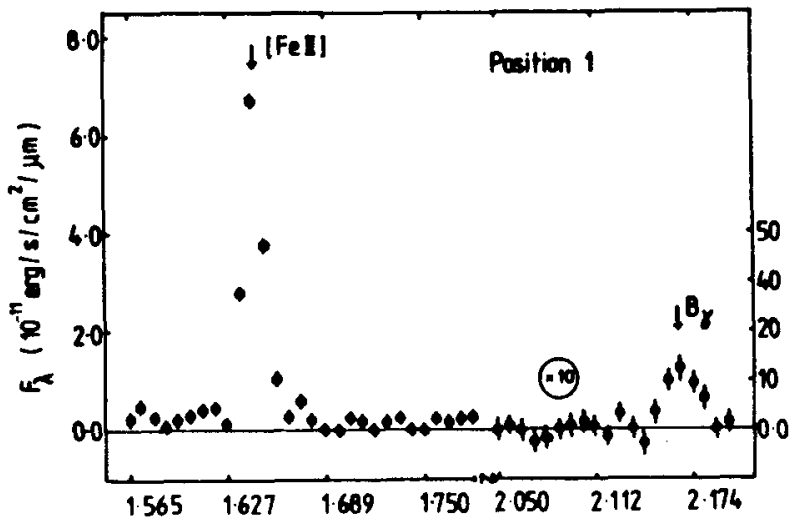

$\lambda(\mu \mathrm{m})$ 


\section{EXCITATION OF [FeII]}

The most prominent feature of the IR spectra is the $1.644 \mu \mathrm{m}$ [Fe II] line. The table gives the ratio $[\mathrm{FeII}] 1.644 \mu \mathrm{m} / \mathrm{Bry}$, (dereddened with $\mathrm{E}_{(\mathrm{B}-\mathrm{V})}=0.992$ )

\section{IR Line ratios}

\begin{tabular}{ll} 
Position & {$[\mathrm{FeII}] 1.644 \mu \mathrm{m} / \mathrm{B} \gamma$} \\
\hline A & $26.9 \pm 2.4$ \\
1 & $41.9 \pm 4.9$ \\
31 & $>71(3 \sigma)$
\end{tabular}

These ratios are remarkably large. In Orion [FeII] $1.644 \mu \mathrm{m} / \mathrm{B} \gamma=0.057$, several orders of magnitude smaller than the measured values in IC443. Clearly the [FeII] line is much brighter relative to hydrogen recombination lines in this SNR shock than in an HII region. This could be due to more efficient excitation of Fe II relative to hydrogen recombination, or due to reduced depletion of $\mathrm{Fe}$ in grains, or most likely a combination of both.

The shock models of McKee et al. (1984) can be used to predict the ratio [FeII] $1.644 \mu \mathrm{m} / \mathrm{B} \gamma$. For a preshock density of $10 \mathrm{~cm}^{-3}$, the ratio [FeII] $1.644 \mu \mathrm{m} / \mathrm{B} \gamma$ is 32 and 69 for shock velocities of 40 and $100 \mathrm{~km} / \mathrm{s}$ respectively. Thus the observed [FeII] $1.644 \mu \mathrm{m} / \mathrm{B} \gamma$ ratio is comparable to the calculated values for these conditions. Optical [FeII] lines of moderately low excitation are also present in the spectrum of IC443. However no lines of multiplets which might feed the upper state of the $1.644 \mu \mathrm{m}$ line $\left(a^{4} \mathrm{D}\right)$, such as $a^{4} D-b^{4} F$ or $a^{4} D-b^{2} P$ have been detected. These facts confirm that the excited states of FeIl are populated collisionally.

The [FeII] lines which have been detected arise from upper states with a large range of excitation temperature $(8,000-25,000 \mathrm{~K})$, so the line ratios should be sensitive to temperature. The mean temperature derived from the IR/optical [Fe II] line ratios, $5,100 \pm 1,000 \mathrm{~K}$, is significantly lower than that inferred by Fesen \& Kirshner (1980) from [O III] $(25,000 \mathrm{~K})$, [N II], [SII], or [O II] $(11,000 \mathrm{~K})$. The [Fe II] lines must be excited downstream of the $\mathrm{N}, \mathrm{O}$, and $\mathrm{S}$ lines where the gas is cooler and the ionization lower. There is only a small scatter in the derived temperatures $(-1,600 \mathrm{~K})$ as expected for collisional population. However, there is a significant trend for the temperature derived from different [ $\mathrm{Fe}$ II] lines to correlate with the excitation energy of the upper level of the line, indicating a range of temperature in the $\mathrm{Fe} I I$ ionization zone. The temperature derived from some of the lower excitation [ $\mathrm{Fe}$ II] lines indicates that these lines are excited where hydrogen has recombined $(\mathrm{T}<5000 \mathrm{~K})$. In the shock models of McKee et al. (1984) some emission from $\mathrm{Fe}$ II is excited in the $\mathrm{H}$ recombination zone , but substantial emission is excited in predominantly neutral gas. where ionization of $\mathrm{Fe} \mathrm{II} \mathrm{is} \mathrm{maintained} \mathrm{by} \mathrm{UV}$ photons penetrating far downstream $\left(\sim 10^{15} \mathrm{~cm}\right)$ from the hot postshock gas where they were emitted.

\section{GRAIN DESTRUCTION}

Iron is one of the more highly depleted elements in the interstellar medium, and so predicted $[\mathrm{FeII}]$ intensities are especially sensitive to modification of elemental depletions by dust grain destruction. If we assume that the shock model calculated for $n=10 \mathrm{~cm}^{-3}$ and $\mathrm{v}=100 \mathrm{~km} / \mathrm{s}$ is appropriate for position 1 (optical spectroscopy of this filament indicates $\mathrm{n}=$ $10-20 \mathrm{~cm}^{-3}, \mathrm{v}=65-100 \mathrm{~km} / \mathrm{s}$ ) then the [FeI] $/ \mathrm{Br} \gamma$ ratio requires that $\sim 30 \%$ of the iron bearing grains entering the shock must have been destroyed.

\section{THE MOLECULAR HYDROGEN DETECTION}

Our detection of $\left[\mathrm{Fe} \mathrm{III]} \mathrm{and} \mathrm{H}_{2}\right.$ in the same aperture at position 31 is noteworthy because $\mathrm{Fe} I \mathrm{II}$ and $\mathrm{H}_{2}$ cannot coexist in substantial quantities. As the observed emission arises from the very edge of the remnant it seems unlikely that the [Fe II] and $\mathrm{H}_{2}$ lines arise 
from two distant regions along the same line of sight. The emitting regions are probably separated by a distance less than or comparable to the projected diameter of the aperture on IC443 $(0.14 \mathrm{pc})$. It is possible that the observed emission arises from the SNR shock propagating into two adjacent regions of different density. Alternatively molecules must form downstream of the ionized gas where the [Fe II] lines are excited.

This work was carried out at UKIRT which is operated by the Royal Observatory Edinburgh on behalf of the U.K. Science and Engineering Research Council. J.R.G is supported by fellowships awarded by the Royal Commission for the Exhibition of 1851, and the U.K. Science and Engineering Research Council. This work was also supported in part by the US Department of Energy under contract No. DE-AC 03-76SF00098.

\section{REFERENCES}

Fesen, R. A., and Kirshner R. P. 1980, Ap. J., 242, 1023.

Graham, J. R., Wright, G. S., \& Longmore, A. J. 1987, Ap. J., 313, 847.

Hester, J. J., 1987, B.A.A.S., 18, No. 4, 30.11.

McKee, C. F., Chernoff, D. F., and Hollenbach , D. J. 1984, Galactic and Extragalactic Infrared Spectroscopy, p103. Eds. M.F. Kessler \& J.P. Phillips. D. Reidel, Dordrecht. 